\title{
Tarifbindung und betriebliche Interessenvertretung: Ergebnisse aus dem IAB-Betriebspanel 2018
}

In diesem Beitrag werden - wie auch in den Vorjahren - aktuelle Ergebnisse zur Tarifbindung und der betrieblichen Interessenvertretung präsentiert. Die Befunde basieren auf den jüngst erhobenen Daten des IAB-Betriebspanels des Instituts für Arbeitsmarkt- und Berufsforschung (IAB), das als einzige repräsentative Datenquelle entsprechende Auskünfte in einer langen Zeitreihe für die Gesamtwirtschaft liefert. ${ }^{1}$

PETER ELLGUTH, SUSANNE KOHAUT

\section{Einleitung}

Zwei Säulen tragen das deutsche System der Arbeitsbeziehungen: die Tarifautonomie und die betriebliche Mitarbeitervertretung durch Betriebs- und Personalräte. Überbetriebliche Verbands- oder Flächentarifverträge spielen eine wesentliche Rolle bei der Regelung von Arbeitsbedingungen und bei der Lohnfindung. Sie werden meist für Regionen und Branchen ausgehandelt und sorgen dort für einheitliche Wettbewerbsbedingungen bei den Arbeitskosten. Für den einzelnen Betrieb ergibt sich daraus eine gesicherte Planungs- und Kalkulationsgrundlage. Zudem herrscht Betriebsfrieden während der Laufzeit der Verträge. Der Verhandlungs- und Koordinationsaufwand bei Tarifverhandlungen liegt bei den Verbänden, was die Betriebe zusätzlich entlastet.

Löhne und Arbeitsbedingungen können jedoch nicht nur auf Branchenebene über (Flächen-)Tarifverträge, sondern auch auf Betriebs- oder Unternehmensebene (Firmentarifverträge) oder in individuellen Arbeitsverträgen geregelt werden. Individuelle Arbeitsverträge werden vor allem in kleineren Betrieben geschlossen. Für größere Firmen wird der Verwaltungsaufwand schnell zu groß, wenn mit jedem Beschäftigten einzeln ein Arbeitsvertrag verhandelt werden muss. Für größere Betriebe sind deshalb Firmentarifverträge eine interessante Alternative.
Im Arbeitsrecht haben Tarifverträge Vorrang gegenüber Betriebsvereinbarungen und Einzelarbeitsverträgen und können deshalb auch als Mindestarbeitsbedingungen interpretiert werden. Allerdings gelten Branchentarifverträge ihren Kritikern häufig als zu starr, da sie branchenweit zwischen Gewerkschaften und Arbeitgeberverbänden ausgehandelt werden und somit nicht die Situation jedes einzelnen Betriebs berücksichtigen können. Mittlerweile sind jedoch Öffnungsklauseln in Branchentarifverträgen weit verbreitet, die explizit betriebliche Gestaltungsmöglichkeiten anbieten. Damit haben betriebsbezogene Regelungen und die betrieblichen Interessenvertretungen an Bedeutung gewonnen. Das gesamte Lohnfindungssystem ist vielschichtiger geworden.

Auf betrieblicher Ebene regelt das Betriebsverfassungsgesetz (BetrVG) die institutionalisierte Interessenvertretung der Beschäftigten. Formal herrscht eine klare funktionale Trennung zwischen der Mitbestimmung auf Branchenebene und auf betrieblicher Ebene. Die Praxis ist jedoch geprägt von einem vielfältigen Ineinandergreifen beider Ebenen mit wechselseitigen Abhängigkeiten. Betriebsräten obliegt die Umsetzung und Überwachung geltender Tarifverträge.

\footnotetext{
Dieser Beitrag ist eine Zweitveröffentlichung. Die Ergebnisse sind vorab im IAB-Forum online erschienen (Ellguth 2019; Kohaut 2019)
} 
In diesem Beitrag wollen wir, wie alljährlich an dieser Stelle, aktuelle Ergebnisse zur Tarifbindung und der betrieblichen Interessenvertretung präsentieren. Die Ergebnisse für 2018 beruhen auf Angaben von rund 15300 Betrieben. Bei der Datenauswertung und -präsentation werden nicht einzig die Werte für Gesamtdeutschland ausgewiesen, sondern auch die Differenzen zwischen den östlichen und westlichen Landesteilen, die bei dieser Thematik zum Teil erheblich sind. Aufgrund des Aufbaus der Zufallsstichprobe sind die Ergebnisse repräsentativ für die rund 2,1 Mio. Betriebe in Deutschland mit mindestens einem sozialversicherungspflichtig Beschäftigten. Insgesamt sind in diesen Betrieben etwa 40,6 Mio. Personen beschäftigt. ${ }^{2}$

\section{Entwicklung und Ausmaß der Tarifbindung}

Seit Beginn der Erhebung 1996 bis Mitte der 2oooer Jahre zeigt die Branchentarifbindung in den alten wie in den neuen Bundesländern eine stark rückläufige Tendenz. In Westdeutschland folgt danach zunächst eine Phase der Stabilisierung, die bis 2010 andauerte, während in Ostdeutschland die Reichweite der Tarifbindung stetig wenn auch nur in sehr kleinen Schritten - abnahm. Auch in Westdeutschland ist nach einer „ruhigen“ Phase erneut ein Rückgang zu verzeichnen. In Ostdeutschland wiederum ist in den letzten Jahren eine gewisse Stabilisierung eingetreten mit leichten Zugewinnen am aktuellen Rand.

Abbildung 1 zeigt sowohl die Entwicklung der Flächentarifbindung in der Gesamtwirtschaft wie auch die der Privatwirtschaft. Betrachtet man zunächst die Gesamtwirtschaft, so ging die Flächentarifbindung bezogen auf die Beschäftigten von 1996 bis 2018 in beiden Landesteilen um 21 Prozentpunkte zurück. Nahezu die gleiche Entwicklung ist zu beobachten, wenn nur die Privatwirtschaft betrachtet wird.

Im Folgenden sind aktuelle Ergebnisse des IAB-Betriebspanels für das Jahr 2018 dargestellt, wobei zwischen der Tarifbindung der Betriebe und der Tarifbindung der Beschäftigten unterschieden wird. Betrachtet man zunächst die Betriebe, so zeigt sich, dass hochgerechnet rund $27 \%$ der westdeutschen, aber nur $17 \%$ der ostdeutschen Betriebe durch Branchentarifverträge gebunden waren. Haus- oder Firmentarifverträge galten für $2 \%$ der Betriebe in den alten und etwa $3 \%$ der Betriebe in den neuen Bundesländern (Tabelle 1). Der Rest, also etwa $71 \%$ der westdeutschen und sogar $80 \%$ der ostdeutschen Betriebe, war nicht tarifgebunden. Etwa $42 \%$ der nicht tarifgebundenen Betriebe in Westdeutschland und $36 \%$ in Ostdeutschland gaben an, sich in ihren Einzelarbeitsverträgen an bestehenden Branchentarifen zu orientieren. Allerdings lehnt sich nur ein Teil dieser Betriebe auch in allen relevanten

\section{ABBILDUNG 1}

\section{Flächentarifbindung der Beschäftigten, 1996-2018}

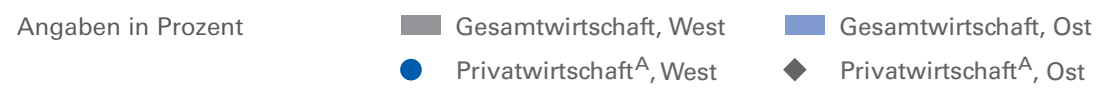

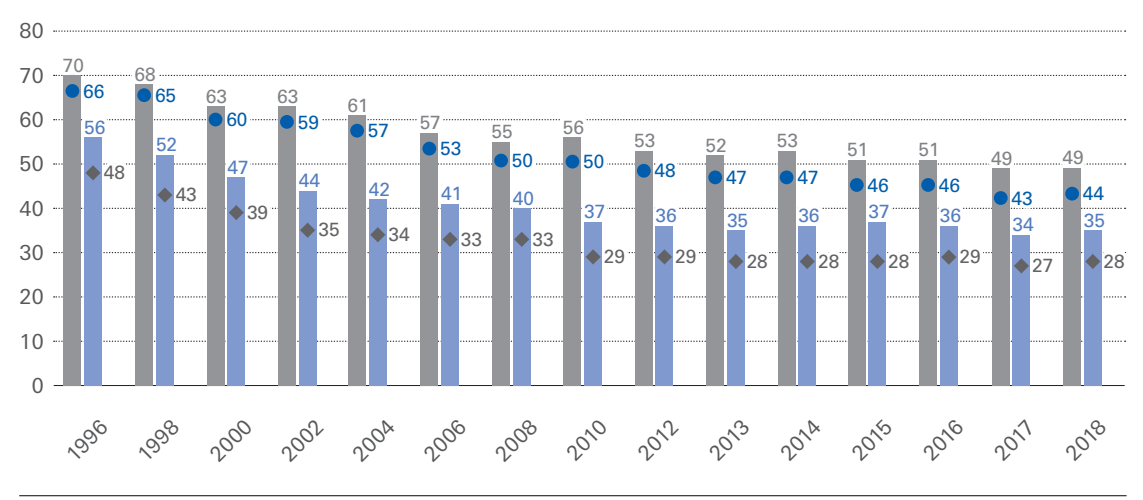

A ohne Landwirtschaft und Organisationen ohne Erwerbszweck

Anmerkung: seit 2010 verändertes Datenprüfverfahren, siehe Ellguth/Kohaut (2011, S. 243)

Quelle: IAB-Betriebspanel 2018, eigene Darstellung

Punkten an den jeweiligen Branchentarif an. Nur in diesen Betrieben dürften die Beschäftigten Arbeitsbedingungen vorfinden, die mit denen in branchentarifgebundenen Betrieben vergleichbar sind. ${ }^{3}$

Ein ähnliches Bild, aber ein größerer Geltungsbereich ergibt sich, wenn statt der Betriebe die Beschäftigten betrachtet werden. So haben im Jahr 2018 hochgerechnet rund $49 \%$ der westdeutschen und etwa $35 \%$ der ostdeutschen Beschäftigten in einem Betrieb gearbeitet, der einem Branchentarifvertrag unterlag (Tabelle 1). Firmentarifverträge galten für $8 \%$ der westdeutschen und $11 \%$ der ostdeutschen Beschäftigten. Für rund $44 \%$ der westdeutschen und $55 \%$ der ostdeutschen Arbeitnehmerinnen und Arbeitnehmer gab es keinen Tarifvertrag. Rund die Hälfte dieser Beschäftigten in westdeutschen Betrieben und etwa $44 \%$ in ostdeutschen Betrieben wurden jedoch indirekt von Tarifverträgen erfasst, da sich ihre Betriebe nach eigenen Angaben daran orientierten.

Geht man weiter ins Detail und analysiert die Tarifbindung in den einzelnen Wirtschaftszweigen, so zeigt

2 Für weitere Informationen zum IAB-Betriebspanel siehe Ellguth et al. (2014)

3 Im Jahr 2011 wurden die Betriebe ausführlicher dazu befragt, ob sie sich bei den Löhnen oder auch bei anderen Regelungen - etwa bei den finanziellen Zusatzleistungen wie Weihnachts- oder Urlaubsgeld, den Arbeitszeiten oder der Dauer des Jahresurlaubs - nach dem Branchentarif richten. Die Befragung hat gezeigt, dass dies bezogen auf die nicht tarifgebundenen Betriebe nur rund $19 \%$ im Westen und $25 \%$ im Osten waren. 
TABELLE 1

\section{Tarifbindung, 2018}

Angaben in Prozent

\begin{tabular}{|c|c|c|c|c|c|c|}
\hline & \multirow{2}{*}{$\begin{array}{r}\text { Betriebe } \\
\text { West }\end{array}$} & \multicolumn{5}{|c|}{ Beschäftigte } \\
\hline & & Ost & Gesamt & West & Ost & Gesamt \\
\hline Branchentarif & 27 & 17 & 25 & 49 & 35 & 46 \\
\hline Firmen-/Haustarif & 2 & 3 & 2 & 8 & 11 & 8 \\
\hline Kein Tarifvertrag & 71 & 80 & 73 & 44 & 55 & 46 \\
\hline $\begin{array}{l}\text { davon Orientierung } \\
\text { am Branchentarif }\end{array}$ & 42 & 36 & 41 & 52 & 44 & 51 \\
\hline
\end{tabular}

Quelle: IAB-Betriebspanel 2018, eigene Darstellung

sich, dass das Ausmaß der Tarifbindung zwischen den Wirtschaftszweigen stark variiert. Tabelle 2 weist den Anteil der tarifgebundenen Betriebe aus. Tabelle 3 zeigt den Anteil der Beschäftigten, die in tarifgebundenen Betrieben arbeiten. ${ }^{4}$

Im Bereich Energie/Wasser/Abfall und Bergbau, im Baugewerbe sowie in der öffentlichen Verwaltung sind überdurchschnittlich viele Betriebe an einen Branchenoder Haustarif gebunden. In diesen Branchen liegt der Anteil der Beschäftigten, die in tarifgebundenen Betrieben arbeiten, weit über dem Durchschnitt. Gleiches gilt für die Finanz- und Versicherungsdienstleistungen. Im Gegensatz hierzu spielen im Bereich der Information und Kommunikation Branchentarifverträge eine nur geringe Rolle.

Betrachtet man die Tarifbindung der Betriebe nach ihrer Größe (Tabelle 4), so ist offensichtlich, dass der Deckungsgrad mit zunehmender Größe steigt. Für beide Landesteile gilt, dass die Branchentarifverträge für Kleinbetriebe eine untergeordnete Rolle spielen, während große Betriebe mit über 200 Beschäftigten in Westdeutschland und mit mehr als 500 Beschäftigten in Ostdeutschland in der Mehrheit tarifgebunden sind. Ebenso nimmt die Bedeutung der Haus- bzw. Firmentarifverträge mit steigender Betriebsgröße zu. Insbesondere in den ostdeutschen Großbetrieben spielen Firmentarifverträge eine große Rolle.

Die Daten des IAB-Betriebspanels lassen sich auch auf Ebene der Bundesländer ${ }^{5}$ auswerten (Tabelle 5). Der Anteil der Beschäftigten in tarifgebundenen Betrieben in den westdeutschen Ländern variiert zwischen rund $40 \%$ in Bremen und $52 \%$ in Nordrhein-Westfalen. Zwischen den ostdeutschen Ländern sind die Unterschiede geringer. Hier liegt die geringste Durchdringung bei $32 \%$ in Sachsen und die stärkste bei rund $39 \%$ in Sachsen-Anhalt sowie Mecklenburg-Vorpommern. Auffallend ist dabei, dass selbst der niedrigste Anteil Beschäftigter in tarifgebundenen Betrieben in einem westdeutschen Bundesland immer noch knapp oberhalb der Werte der ostdeutschen Länder liegt.

\section{Aktuelle Ergebnisse zur betrieblichen Interessenvertretung}

Das bundesdeutsche System der Interessenvertretung mit seiner dualen Struktur von sektoral ausgehandelten Tarifverträgen und betrieblicher Interessenregulierung ist seit Mitte der 1980er Jahre durch eine Verlagerung von Regelungskompetenzen von der überbetrieblichen auf die betriebliche Ebene gekennzeichnet. In der wissenschaftlichen und politischen Debatte wird diese Bedeutungsverschiebung in erster Linie unter dem Aspekt der Flexibilisierung des Tarifsystems diskutiert. Die in diesem Zusammenhang nach wie vor geforderte Erweiterung betrieblicher Handlungsspielräume tarifgebundener Betriebe setzt (mehr oder weniger stillschweigend) die Existenz eines kompetenten betrieblichen Verhandlungspartners voraus, der die Interessen der Arbeitnehmer bündelt und vertritt. So wird z. B. in vielen tariflichen Öffnungsklauseln, die für die Betriebe die Möglichkeit vorsehen, von den tariflichen Standards nach unten abzuweichen, explizit auf die diesbezügliche Gestaltungskompetenz des Betriebsrats verwiesen. Aber auch - oder gerade - für die Betriebe ohne Tarifbindung stellt sich die Frage nach einer (gesetzlich legitimierten) Vertretung der Beschäftigten auf betrieblicher Ebene.

Vor diesem Hintergrund soll zunächst analog zur Tarifbindung ein Eindruck der quantitativen Reichweite der betrieblichen Mitbestimmung, d.h. der Verbreitung von Betriebsräten vermittelt werden. In Anbetracht des zunehmenden Interesses an den Arbeitsbeziehungen in betriebsratslosen Betrieben werden ergänzend auch Zahlen zu betriebsspezifischen Formen der Mitarbeitervertretung, wie sie seit 2004 im IAB-Betriebspanel erhoben werden, dargestellt. Der Frage, inwieweit diese Einrichtungen eine funktionales Äquivalent zum Betriebsrat sein können, kann an dieser Stelle allerdings nicht nachgegangen werden (siehe dazu: Ellguth 2009). Abschließend sollen dann beide Ebenen der Tarif- und Mitbestimmungslandschaft gemeinsam betrachtet werden.

In Tabelle 6 sind die aktuellen Daten zur Existenz von Betriebsräten und alternativen Vertretungsformen in der

4 Abweichend zur Berichterstattung in früheren Jahren werden die Branchen- und Haustarifbindung für West- und Ostdeutschland nicht mehr getrennt ausgewiesen, da in vielen Branchen die Anzahl der Betriebe mit Haustarifvertrag in der Stichprobe so niedrig ist, dass die Ergebnisse nicht mehr sinnvoll interpretiert werden können.

5 Die Stichprobe des IAB-Betriebspanels ist so aufgebaut dass die Ergebnisse auch jeweils für die einzelnen Bundesländer repräsentativ sind. Nur für Hamburg genügt die Fallzahl für eine gesonderte Auswertung nicht. Aus diesem Grund wurden Hamburg und Schleswig-Holstein zusammengefasst. 
TABELLE 2

Tarifbindung der Betriebe nach Wirtschaftszweig, 2018

Angaben in Prozent

\begin{tabular}{|c|c|c|c|c|c|c|c|c|c|c|}
\hline & Gesamtdeut & chland & & & Westdeutsc & & & Ostdeutschl & & \\
\hline & $\begin{array}{l}\text { Branchen- } \\
\text { tarif }\end{array}$ & Haustarif & Kein Tarif & $\begin{array}{l}\text { davon Ori- } \\
\text { entierung } \\
\text { am Tarif }\end{array}$ & $\begin{array}{l}\text { Branchen- } \\
\text { oder } \\
\text { Haustarif }\end{array}$ & Kein Tarif & $\begin{array}{l}\text { davon Ori- } \\
\text { entierung } \\
\text { am Tarif }\end{array}$ & $\begin{array}{l}\text { Branchen- } \\
\text { oder } \\
\text { Haustarif }\end{array}$ & Kein Tarif & $\begin{array}{l}\text { davon Ori- } \\
\text { entierung } \\
\text { am Tarif }\end{array}$ \\
\hline Landwirtschaft u. a. & 23 & (0) & 77 & 39 & 27 & 73 & 41 & 9 & 91 & 29 \\
\hline Energie/Wasser/Abfall und Bergbau & 48 & 11 & 41 & 54 & 64 & 36 & (56) & 41 & 59 & 49 \\
\hline Verarbeitendes Gewerbe & 22 & 3 & 75 & 53 & 28 & 73 & 57 & 14 & 86 & 40 \\
\hline Baugewerbe & 47 & 1 & 52 & 52 & 50 & 50 & 53 & 42 & 58 & 49 \\
\hline Großhandel, KfZ-Handel und -reparatur & 20 & 2 & 78 & 47 & 24 & 77 & 50 & 13 & 87 & 36 \\
\hline Einzelhandel & 20 & 2 & 78 & 41 & 24 & 76 & 42 & 14 & 86 & 40 \\
\hline Verkehr und Lagerei & 20 & 4 & 76 & 34 & 28 & 72 & 32 & 6 & 94 & 38 \\
\hline Information und Kommunikation & 5 & (1) & 94 & 26 & 7 & 93 & 28 & (4) & 96 & (21) \\
\hline Finanz- und Versicherungsdienstleistungen & 26 & (1) & 74 & 37 & 28 & 73 & 38 & 21 & 79 & (33) \\
\hline Gastgewerbe und Sonstige Dienstleistungen & 23 & 1 & 77 & 34 & 26 & 74 & 35 & 13 & 87 & 29 \\
\hline Gesundheit und Erziehung/Unterricht & 29 & 5 & 66 & 53 & 37 & 63 & 55 & 22 & 78 & 48 \\
\hline $\begin{array}{l}\text { Wirtschaftliche, wissenschaftliche } \\
\text { und freiberufliche Dienstleistungen }\end{array}$ & 17 & 1 & 83 & 30 & 18 & 82 & 31 & 16 & 84 & 23 \\
\hline Organisationen ohne Erwerbscharakter & 27 & 12 & 61 & 42 & 41 & 59 & 45 & 31 & 69 & 35 \\
\hline Öffentliche Verwaltung/Sozialversicherung & 85 & 6 & 9 & (44) & 91 & 9 & (41) & 89 & (11) & (52) \\
\hline Gesamt & 25 & 2 & 73 & 41 & 29 & 71 & 42 & 20 & 80 & 36 \\
\hline
\end{tabular}

Werte in Klammern wenig belastbar, da weniger als 20 Fälle in der Stichprobe

Quelle: IAB-Betriebspanel 2018, eigene Darstellung

TABELLE 3

Beschäftigte in tarifgebundenen Betrieben nach Wirtschaftszweig, 2018

Angaben in Prozent

\begin{tabular}{|c|c|c|c|c|c|c|c|c|c|c|}
\hline & Gesamtdeuts & chland & & & Westdeutsc & & & Ostdeutschla & & \\
\hline & $\begin{array}{c}\text { Branchen- } \\
\text { tarif }\end{array}$ & Haustarif & Kein Tarif & $\begin{array}{l}\text { davon Ori- } \\
\text { entierung } \\
\text { am Tarif }\end{array}$ & $\begin{array}{l}\begin{array}{c}\text { Branchen- } \\
\text { oder } \\
\text { Haustarif }\end{array}\end{array}$ & Kein Tarif & $\begin{array}{l}\text { davon Ori- } \\
\text { entierung } \\
\text { am Tarif }\end{array}$ & $\begin{array}{l}\text { Branchen- } \\
\text { oder- } \\
\text { Haustarif }\end{array}$ & Kein Tarif & $\begin{array}{l}\text { davon Ori- } \\
\text { entierung } \\
\text { am Tarif }\end{array}$ \\
\hline Landwirtschaft u. a. & 36 & (2) & 63 & 46 & 41 & 59 & 50 & 25 & 75 & 37 \\
\hline Energie/Wasser/Abfall und Bergbau & 63 & 18 & 18 & 59 & 85 & 15 & (54) & 70 & 30 & 68 \\
\hline Verarbeitendes Gewerbe & 45 & 11 & 44 & 61 & 59 & 41 & 63 & 33 & 67 & 51 \\
\hline Baugewerbe & 60 & 2 & 38 & 63 & 64 & 37 & 65 & 56 & 44 & 54 \\
\hline Großhandel, KfZ-Handel und -reparatur & 32 & 4 & 64 & 52 & 38 & 62 & 55 & 24 & 76 & 37 \\
\hline Einzelhandel & 30 & 6 & 64 & 58 & 38 & 62 & 60 & 25 & 75 & 50 \\
\hline Verkehr und Lagerei & 35 & 16 & 49 & 42 & 54 & 46 & 42 & 35 & 65 & 43 \\
\hline Information und Kommunikation & 11 & (9) & 80 & 20 & 18 & 82 & 22 & (29) & 71 & (8) \\
\hline Finanz- und Versicherungsdienstleistungen & 78 & (3) & 20 & 42 & 82 & 18 & 44 & 71 & 29 & (32) \\
\hline Gastgewerbe und Sonstige Dienstleistungen & 37 & 2 & 60 & 39 & 41 & 59 & 40 & 33 & 67 & 36 \\
\hline Gesundheit und Erziehung/Unterricht & 47 & 13 & 41 & 62 & 62 & 38 & 63 & 48 & 52 & 58 \\
\hline $\begin{array}{l}\text { Wirtschaftliche, wissenschaftliche } \\
\text { und freiberufliche Dienstleistungen }\end{array}$ & 45 & 5 & 49 & 36 & 52 & 48 & 38 & 46 & 54 & 30 \\
\hline Organisationen ohne Erwerbscharakter & 46 & 12 & 41 & 62 & 64 & 36 & 63 & 38 & 62 & 62 \\
\hline Öffentliche Verwaltung/Sozialversicherung & 88 & 10 & 3 & (54) & 97 & 3 & (49) & 99 & (1) & (91) \\
\hline Gesamt & 46 & 8 & 46 & 51 & 56 & 44 & 52 & 45 & 55 & 44 \\
\hline
\end{tabular}

Werte in Klammern wenig belastbar, da weniger als 20 Fälle in der Stichprobe

Quelle: IAB-Betriebspanel 2018, eigene Darstellung 
Tarifbindung der Betriebe nach Betriebsgröße, 2018

Angaben in Prozent

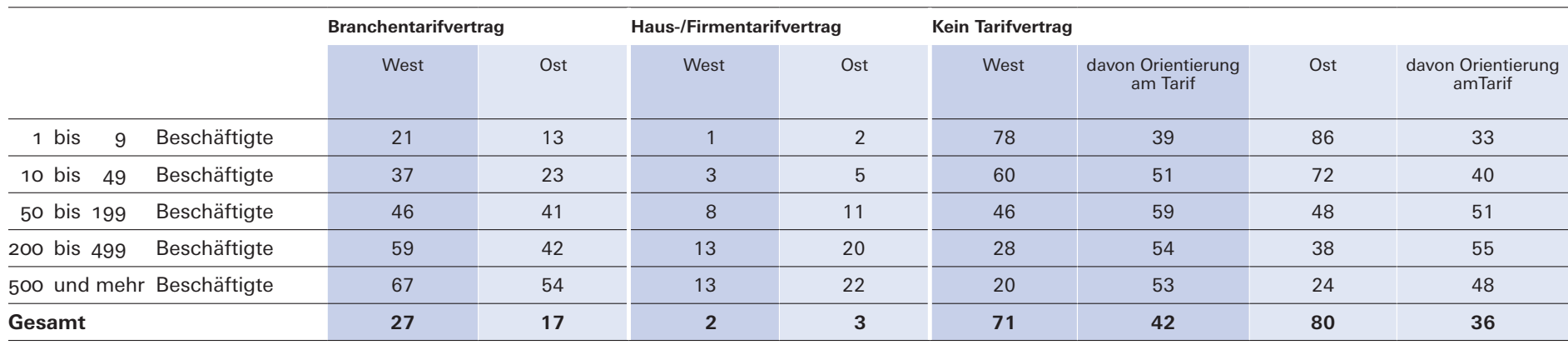

Privatwirtschaft (Betriebe ab fünf Beschäftigte) für Westund Ostdeutschland dargestellt. Für den Anteil der Betriebe mit gesetzlich legitimierter Interessenvertretung wird mit $9 \%$ im Westen das Niveau der letzten Jahre bestätigt. Im Osten wird der Vorjahreswert um einen Prozentpunkt gesteigert.

Bei der letztjährigen Berichterstattung hatten wir in der Gesamtschau der vergangenen gut 20 Jahre einen Tiefstand des auf Beschäftigte bezogenen Deckungsgrads

TABELLE 5

\section{Anteil der Beschäftigten in tarifgebundenen Betrieben nach Bundesland, 2018}

Angaben in Prozent

\begin{tabular}{lccc}
\hline & Branchentarif & Haustarif & kein Tarifvertrag \\
\hline Schleswig-Holstein, Hamburg & 45 & 8 & 47 \\
\hline Niedersachsen & 47 & 13 & 40 \\
\hline Bremen & 40 & 15 & 45 \\
\hline Nordrhein-Westfalen & 52 & 8 & 40 \\
\hline Hessen & 48 & 9 & 43 \\
\hline Rheinland-Pfalz & 48 & 7 & 44 \\
\hline Baden-Württemberg & 45 & 7 & 48 \\
\hline Bayern & 49 & 5 & 46 \\
\hline Saarland & 50 & 7 & 44 \\
\hline Berlin & 33 & 13 & 54 \\
\hline Brandenburg & 35 & 14 & 51 \\
\hline Mecklenburg-Vorpommern & 39 & 8 & 53 \\
\hline Sachsen & 32 & 9 & 60 \\
\hline Sachsen-Anhalt & 39 & 11 & 50 \\
\hline Thüringen & 34 & 11 & 55 \\
\hline Insgesamt & $\mathbf{4 6}$ & $\mathbf{8}$ & $\mathbf{4 6}$ \\
\hline
\end{tabular}

Quelle: IAB-Betriebspanel 2018, eigene Darstellung in beiden Landesteilen konstatiert (Ellguth/Kohaut 2018, S. 303). Dieser Rückgang hat sich bis zur Jahresmitte 2018 nicht fortgesetzt. Hier werden mit $35 \%$ und $42 \%$ (Ost-/ Westdeutschland) die Vorjahreswerte um jeweils zwei Prozentpunkte übertroffen. Somit kann von einer gewissen Stabilität der quantitativen Basis der betrieblichen Mitbestimmung am aktuellen Rand gesprochen werden. Ob sich hier gar eine Trendwende ankündigt, muss die weitere Entwicklung zeigen. An dem seit Beginn der Datenerhebung im IAB-Betriebspanel zu beobachtenden deutlichen Niveauunterschied zwischen beiden Landesteilen hat sich auch im vergangenen Jahr kaum etwas geändert.

Die hinlänglich bekannten größenspezifischen Unterschiede in der Verbreitung von Betriebsräten stellen sich ebenfalls wieder ein. Nur jeder 20. Kleinbetrieb (bis 50 Beschäftigte) in West- und jeder 16. in Ostdeutschland verfügt über einen Betriebsrat. Unter den Großbetrieben (über 500 Beschäftigte) ist die Existenz eines Betriebsrats dagegen die Regel und erreicht in beiden Landesteilen einen Anteil von knapp 90\%. Prägt in betrieblicher Perspektive die große Zahl der Kleinbetriebe das Gesamtbild, so relativiert sich deren Gewicht natürlich bei Betrachtung des auf Beschäftigte bezogenen Deckungsgrads. Die insgesamt um rund 7 Prozentpunkte niedrigere Reichweite der betrieblichen Mitbestimmung in Ostdeutschland resultiert vor allem aus der geringeren Verbreitung von Betriebsräten im mittelbetrieblichen Segment.

Ebenso bestätigen sich die erwarteten starken Unterschiede zwischen einzelnen Branchen, und das sowohl in Betriebs- als auch Beschäftigtenperspektive (Tabelle 7). Die größte quantitative Reichweite herrscht im traditionell stark mitbestimmten Bereich Energie- und Wasserversorgung/Abfallwirtschaft/Bergbau, in den Finanz- und Versicherungsdienstleistungen und im Verarbeitenden Gewerbe. Das untere Ende bilden die vorwiegend kleinbetrieblich strukturierten Branchen des sonstigen Dienstleitungsbereichs (inkl. Gastgewerbe) und der Bauwirtschaft. 
TABELLE 6

Betriebsrat und andere Formen der Mitarbeitervertretung nach Betriebsgröße, $2018^{\mathrm{A}}$

Angaben in Prozent

\begin{tabular}{|c|c|c|c|c|c|c|c|c|c|}
\hline & & \multicolumn{4}{|c|}{ Betriebsgrößenklassen (Anzahl Beschäftigte) } & \multirow[b]{3}{*}{501 u.m. } & \multirow{3}{*}{$\begin{array}{l}\text { Insgesamt } \\
\quad(\text { ab 5) }\end{array}$} & & \\
\hline & & & & & & & & \multicolumn{2}{|c|}{ nachrichtlich: } \\
\hline & & $5-50$ & $51-100$ & $101-199$ & $200-500$ & & & $a b 21$ & $a b 51$ \\
\hline & & \multicolumn{8}{|c|}{ Westdeutschland } \\
\hline \multirow[t]{2}{*}{ Betriebe } & \multirow{2}{*}{$\begin{array}{l}\text { mit } B R \\
\text { mit anderer MA-Vertretung }\end{array}$} & 5 & 32 & 53 & 73 & 87 & 9 & 26 & 46 \\
\hline & & 18 & 21 & 16 & 16 & 16 & 18 & 22 & 18 \\
\hline \multirow[t]{2}{*}{ Beschäftigte } & \multirow{2}{*}{$\begin{array}{l}\text { mit BR } \\
\text { mit anderer MA-Vertretung }\end{array}$} & 8 & 33 & 54 & 73 & 90 & 42 & 55 & 67 \\
\hline & & 21 & 21 & 16 & 16 & 22 & 20 & 20 & 19 \\
\hline & & \multicolumn{8}{|c|}{ Ostdeutschland } \\
\hline \multirow[t]{2}{*}{ Betriebe } & \multirow{2}{*}{$\begin{array}{l}\text { mit } B R \\
\text { mit anderer MA-Vertretung }\end{array}$} & 6 & 36 & 45 & 69 & 88 & 10 & 26 & 44 \\
\hline & & 9 & 15 & 19 & 28 & 22 & 10 & 15 & 18 \\
\hline \multirow[t]{2}{*}{ Beschäftigte } & \multirow{2}{*}{$\begin{array}{l}\text { mit } B R \\
\text { mit anderer MA-Vertretung }\end{array}$} & 10 & 37 & 45 & 72 & 86 & 35 & 47 & 58 \\
\hline & & 11 & 14 & 19 & 27 & 25 & 16 & 19 & 21 \\
\hline
\end{tabular}

A Basis: privatwirtschaftliche Betriebe ab 5 Beschäftigte, ohne Landwirtschaft und Organisationen ohne Erwerbszweck

Quelle: IAB-Betriebspanel 2018, eigene Darstellung

TABELLE 7

Betriebsrat und andere Formen der Mitarbeitervertretung nach Branche, 2018 ${ }^{\mathrm{A}}$

Angaben in Prozent

\begin{tabular}{|c|c|c|c|c|c|c|c|c|c|c|c|c|}
\hline & & \multicolumn{6}{|c|}{ Wirtschaftszweige (Gesamtdeutschland) } & \multirow[b]{2}{*}{ 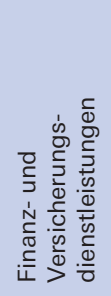 } & \multirow[b]{2}{*}{ 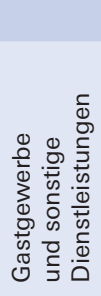 } & \multirow[b]{2}{*}{ 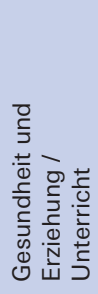 } & \multirow[b]{2}{*}{ 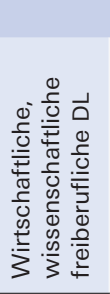 } & \multirow[b]{2}{*}{ 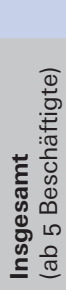 } \\
\hline & & 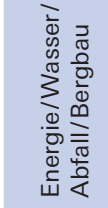 & 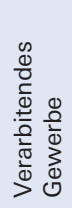 & 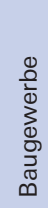 & $\begin{array}{l}\overline{0} \\
\frac{0}{0} \\
\frac{1}{\pi} \\
\text { I }\end{array}$ & 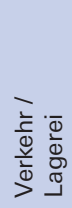 & 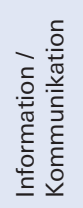 & & & & & \\
\hline \multirow[t]{2}{*}{ Betriebe } & \multirow{2}{*}{$\begin{array}{l}\text { mit } B R \\
\text { mit anderer MA-Vertretung }\end{array}$} & 40 & 16 & 2 & 9 & 10 & 9 & 21 & 3 & 14 & 7 & 9 \\
\hline & & 14 & 15 & 16 & 14 & 14 & 19 & 18 & 15 & 23 & 16 & 16 \\
\hline \multirow[t]{2}{*}{ Beschäftigte } & \multirow{2}{*}{$\begin{array}{l}\text { mit } B R \\
\text { mit anderer MA-Vertretung }\end{array}$} & 79 & 65 & 16 & 29 & 41 & 34 & 66 & 11 & 50 & 28 & 41 \\
\hline & & 14 & 18 & 18 & 17 & 18 & 18 & 11 & 18 & 27 & 17 & 19 \\
\hline
\end{tabular}

A Basis: privatwirtschaftliche Betriebe ab 5 Beschäftigte, ohne Landwirtschaft und Organisationen ohne Erwerbszweck

Quelle: IAB-Betriebspanel 2018, eigene Darstellung

Branchenspezifische Entwicklungen lassen sich wegen der 2009 erfolgten Umstellung auf eine neue Wirtschaftszweigsystematik (WZ20o8) nur für einen eingeschränkten Zeitraum verfolgen. Klar identifizierbar sind für die vergangenen neun Jahre Rückgänge in den Finanz- und Versicherungsdienstleistungen und im Bereich Verkehr/ Lagerei, also in Branchen, die sich vormals eher am oberen Ende der Verteilung fanden.

Analog zur Tarifbindung soll auch die Reichweite von Betriebsräten im Vergleich der einzelnen Bundesländer dargestellt werden. Für die Geltung eines Tarifvertrags war deutlich geworden, dass der Niveauunterschied zwischen West- und Ostdeutschland auf länderspezifischen Abstufungen der Tarifbindung beruht, wobei die westli- chen Bundesländer insgesamt eine höhere Tarifbindung ausweisen. Genauer: Das ostdeutsche Bundesland mit der höchsten Tarifbindung reicht nicht an das westliche Bundesland mit der niedrigsten Tarifbindung heran. Beim Anteil der Beschäftigten in Betrieben mit Betriebsrat ergibt sich ein nicht ganz so eindeutiges Gefälle zwischen den beiden Landesteilen.

In den westdeutschen Bundesländern rangiert der Anteil der Beschäftigten in Betrieben mit Betriebsrat zwischen $48 \%$ und $32 \%$, in den ostdeutschen zwischen $37 \%$ und $29 \%$ (Tabelle 8). Bis auf Berlin bilden die ostdeutschen Bundesländer eine recht homogene Gruppe mit Werten zwischen $35 \%$ und $37 \%$, wobei aber alle Länder klar unter dem bundesdeutschen Durchschnitt liegen. 


\section{Betriebsspezifische Formen der Mitarbeitervertretung}

Alternativ zu oder auch neben einem Betriebsrat kann es in den Betrieben andere betriebsspezifische Formen der Mitarbeitervertretung geben. Die folgenden Angaben beziehen sich ebenfalls auf die privatwirtschaftlichen Betriebe $\mathrm{ab}$ fünf Beschäftigte. Die Größenabgrenzung wurde gewählt, um eine Gegenüberstellung mit den Zahlen zur Verbreitung eines Betriebsrats zu ermöglichen. Zudem spielen solche Einrichtungen in den Kleinstbetrieben ohnehin so gut wie keine Rolle. Das gerade angesprochene Nebeneinander von Betriebsrat und "hausgemachter" Mitarbeitervertretung gibt es allerdings nur bei einem Anteil von ca. $1 \%$ der Betriebe (ohne Tabelle). Wie nicht anders zu erwarten, kommt dies aber in größeren Betrieben deutlich häufiger vor (bei ca. $11 \%$ der Betriebe mit mehr als 500 Beschäftigten).

Die Verbreitung solcher Einrichtungen, seien es „Runde Tische“ oder „Belegschaftssprecher“ etc., zeigt nicht annähernd die gleiche Stabilität, wie dies die Institution des Betriebsrats auszeichnet. Von einer Erhebungswelle zur nächsten gibt es z.T. deutliche Schwankungen. In Westdeutschland pendeln die betrieblichen Anteilswerte zwischen $7 \%$ und $18 \%$, in Ostdeutschland zwischen $3 \%$ und $10 \%$. Beide Maximalwerte werden in der aktuellen Erhebungswelle erreicht und markieren einen klaren Bedeutungszuwachs dieser Einrichtungen (Tabelle 6). Wie sich darüber hinaus zeigt, existiert - anders als beim Betriebsrat - eine große Fluktuation bei diesen Gremien, d.h. ein Großteil davon existiert nur über einen relativ kurzen Zeitraum, dafür werden auch viele Jahr für Jahr neu eingerichtet. Betrachtet man z.B. die Betriebe über
TABELLE 8

\section{Anteil der Beschäftigten in Betrieben mit Betriebsrat nach Bundesland, 2018 ${ }^{A}$ \\ Angaben in Prozent}

\begin{tabular}{ll}
\hline Bundesländer & 48 \\
\hline Bremen & 46 \\
\hline Hessen & 44 \\
\hline Niedersachsen & 44 \\
\hline Nordrhein-Westfalen & 44 \\
\hline Schleswig-Holstein/Hamburg & 41 \\
\hline Bayern & 40 \\
\hline Baden-Württemberg & 37 \\
\hline Sachsen-Anhalt & 37 \\
\hline Mecklenburg-Vorpommern & 36 \\
\hline Thüringen & 36 \\
\hline Sachsen & 35 \\
\hline Rheinland-Pfalz & 35 \\
\hline Brandenburg & 32 \\
\hline Saarland & 29 \\
\hline Berlin & 41 \\
\hline Insgesamt & \\
\hline
\end{tabular}

A Basis: privatwirtschaftliche Betriebe ab 5 Beschäftigte, ohne Landwirtschaft und Organisationen ohne Erwerbszweck

Quelle: IAB-Betriebspanel 2018, eigene Darstellung

einen Zeitraum von vier Jahren (2013-2017), verfügen nur $2 \%$ dieser Betriebe mit $4 \%$ der Beschäftigten dauerhaft über eine solche Einrichtung. Ein Betriebsratsgremium hingegen existiert über diesen Zeitraum hinweg in $8 \%$ der überlebenden Betriebe mit $45 \%$ der Beschäftigten (vgl. dazu auch Ellguth 2009, S. 116).

TABELLE 9

\section{Tarifbindung und Betriebsrat, 1996-2018 ${ }^{\mathrm{A}}$}

Anteil der jeweils betroffenen Beschäftigten in Prozent

\begin{tabular}{|c|c|c|c|c|c|c|c|c|c|c|c|c|c|c|c|c|}
\hline & \multicolumn{8}{|c|}{ Westdeutschland } & \multicolumn{8}{|c|}{ Ostdeutschland } \\
\hline & 1996 & 2000 & 2004 & 2008 & 2012 & 2014 & 2016 & 2018 & 1996 & 2000 & 2004 & 2008 & 2012 & 2014 & 2016 & 2018 \\
\hline BR und Branchentarif & 41 & 37 & 35 & 30 & 29 & 28 & 27 & 26 & 29 & 25 & 22 & 18 & 15 & 15 & 14 & 14 \\
\hline BR und Haustarif & \multirow{2}{*}{$9^{B}$} & 6 & 6 & 6 & 6 & 6 & 7 & 7 & \multirow{2}{*}{$13^{B}$} & 8 & 9 & 9 & 11 & 9 & 9 & 8 \\
\hline BR und kein Tarif & & 7 & 6 & 9 & 8 & 9 & 9 & 10 & & 8 & 9 & 10 & 10 & 9 & 11 & 13 \\
\hline Branchentarif und kein BR & 27 & 25 & 24 & 22 & 21 & 21 & 21 & 19 & 22 & 16 & 16 & 18 & 16 & 15 & 17 & 15 \\
\hline Haustarif und kein BR & \multirow{2}{*}{$23^{B}$} & 1 & 1 & 2 & 1 & 2 & 1 & 1 & \multirow{2}{*}{$36^{\mathrm{B}}$} & 3 & 4 & 4 & 3 & 3 & 2 & 2 \\
\hline kein Tarif und kein BR & & 24 & 27 & 31 & 34 & 34 & 36 & 37 & & 39 & 41 & 42 & 45 & 49 & 47 & 48 \\
\hline Gesamt & 100 & 100 & 100 & 100 & 100 & 100 & 100 & 100 & 100 & 100 & 100 & 100 & 100 & 100 & 100 & 100 \\
\hline
\end{tabular}

A ohne Landwirtschaft und Betriebe ohne Erwerbszweck

B Da sich die Abfrage der Geltung eines Haus- bzw. Firmentarifvertrags zwischen 1996 und 1998 geändert hat, können die entsprechenden Werte erst von 1998 an ausgewiesen werden.

Für 1996 werden deshalb die zusammengefassten Werte der Betriebe ohne Branchentarif angegeben 
Die in den alten Bundesländern wesentlich stärkere Verbreitung betriebsspezifischer Mitarbeitervertretungen drückt sich auch in den auf die Beschäftigten bezogen Zahlen aus. Zurzeit arbeiten in Westdeutschland $20 \%$ der Beschäftigten in Betrieben mit einer solchen Einrichtung, in Ostdeutschland sind dies $16 \%$. Anders als beim Betriebsrat gibt es hier keine so klare größenspezifische Verteilung (Tabelle 6). Unterschiede entlang der Branchen sind ebenfalls geringer ausgeprägt als beim Betriebsrat. Im Segment mit der stärksten Verbreitung „Gesundheitswesen, Erziehung und Unterricht" kommen rund $27 \%$ der Beschäftigten in den Genuss einer solchen Einrichtung (Tabelle 7), in den übrigen Branchen bewegen sich die Anteilswerte zwischen $11 \%$ (Finanz- und Versicherungsdienstleistungen) und $18 \%$ (in mehreren Wirtschaftszweigen). Diese Zahlen können natürlich keine Auskunft über die Ausgestaltung der betreffenden Gremien oder gar deren betriebspolitische Rolle geben. Dazu sei auf eine Erhebung von Hauser-Ditz et al. (2006) verwiesen.

\section{Das Zusammenspiel von betrieblicher und überbetrieblicher Interessenvertretung}

Der abschließende gemeinsame Blick auf die betriebliche und sektorale Ebene der (gesetzlich legitimierten) Interessenvertretung soll insbesondere einen Eindruck von den Verschiebungen im dualen System geben. Damit gilt im Folgenden das Augenmerk zum einen der Entwicklung der sogenannten betrieblichen Vertretungslücken. Das sind Betriebe, die zwar tarifgebunden sind, aber über keinen Betriebsrat verfügen; zum anderen geht es um die sogenannten weißen Flecken in der Tarif- und Mitbestimmungslandschaft, wo es keinerlei gesetzlich legitimierte Interessenvertretung gibt, also weder von Gewerkschaftsseite noch auf Betriebsebene. Die präsentierten Zahlen beziehen sich ebenfalls auf die Betriebe der Privatwirtschaft ab fünf Beschäftigte. Eine Berücksichtigung der Kleinstbetriebe, die ja nicht zur Wahl eines Betriebsrats berechtigt sind, würde bei der gemeinsamen Betrachtung des Deckungsgrades von betrieblicher Mitbestimmung und Tarifbindung ein systematisch verzerrtes Bild liefern.

Nur eine Minderheit der in der Privatwirtschaft (ab fünf Beschäftigte) beschäftigten Arbeitnehmer ist in Betrieben tätig, die zur Kernzone des dualen Systems der Interessenvertretung zu zählen sind. Diese Zone umfasst nur etwas mehr als ein Viertel (26\%) der Beschäftigten in Westdeutschland und nur noch $14 \%$ in Ostdeutschland (Tabelle 9). Von einer betrieblichen Vertretungslücke (Betrieb ist an einen Branchentarif gebunden, verfügt aber über keinen Betriebsrat) sind $19 \%$ der Beschäftigten in Westdeutschland und $15 \%$ in Ostdeutschland betroffen. Diese vermeintlich günstigere Situation im Osten ist allerdings nur der dort geringeren Reichweite der Tarifbindung geschuldet, die sich in deutlicher ausgeprägten „weißen Flecken der Tarif- und Mitbestimmungslandschaft“ äußert. Während in Westdeutschland „nur“ $37 \%$ der Beschäftigten ohne Branchentarif und ohne Betriebsrat auskommen müssen, sind dies in Ostdeutschland $48 \%$.

Seit 1996 ist die Kernzone des dualen Systems in beiden Landesteilen deutlich geschrumpft, und zwar um jeweils 15 Prozentpunkte. In fast gleichem Umfang sind die „weißen Flecken in der Tarif- und Mitbestimmungslandschaft" angewachsen, wodurch immer weitere Teile der Be- triebslandschaft ohne jegliche Form der gesetzlich legitimierten Interessenvertretung bleiben. Ein weiteres im Beobachtungszeitraum (vor allem in Ostdeutschland) gewachsenes Segment umfasst Betriebe, die nicht (mehr) an einen Branchentarif gebunden sind, aber über einen Betriebsrat verfügen. Abgenommen hat hingegen in den letzten gut 20 Jahren der Umfang der betrieblichen Vertretungslücken (tarifgebundene Betriebe ohne Betriebsrat). Dies ist aber alles andere als eine Erfolgsmeldung, da die betreffenden Betriebe zumeist aus dieser Gruppe in die Gruppe der Betriebe gewechselt sind, die weder eine Interessenvertretung noch eine Tarifbindung vorzuweisen haben.

Diese Trends sind überwiegend dem Rückgang der Branchentarifbindung geschuldet bei parallel vergleichsweise moderaten Verlusten in der Reichweite der betrieblichen Mitbestimmung. Insgesamt ergibt die jüngste Entwicklung ein eher uneinheitliches Bild mit Veränderungen im Vergleich zum Vorjahr, die z. T. auf eine weitere Erosion (der Tarifbindung in Westdeutschland) hindeuten, z. T. aber auch eine Trendwende (für die Reichweite der betrieblichen Mitbestimmung) markieren könnten.

\section{LITERATUR}

Ellguth, P. (2009): Betriebsspezifische Formen der Mitarbeitervertretung - welche Betriebe, welche personalpolitischen Wirkungen, in: Industrielle Beziehungen 16 (2), S. 109-135

Ellguth, P. (2019): Ist die Erosion der betrieblichen Mitbestimmung gestoppt?, in: IAB-Forum vom 22.05.2019, https://www.iab-forum.de/ist-die-erosion-der-betrieblichen-mitbestimmung-gestoppt

Ellguth, P./ Kohaut, S./ Möller, I. (2014): The IAB-Establishment Panel - methodological essentials and data quality, in: Journal for Labour Market Research 47 (1-2), S. 27-41

Ellguth, P./ Kohaut, S. (2018): Tarifbindung und betriebliche Interessenvertretung. Aktuelle Ergebnisse aus dem IAB-Betriebspanel 2017, in: WSI-Mitteilungen 71 (4), S. 299-306, https://www.boeckler.de/wsi-mitteilungen_115204_115217.htm Ellguth, P./ Kohaut, S. (2011): Tarifbindung und betriebliche Interessensvertretung: Aktuelle Ergebnisse aus dem IAB-Betriebspanel 2010, in: WSI-Mitteilungen 64 (5), S. 242-247, https://www.boeckler.de/wsimit_2011_05_ellguth.pdf Hauser-Ditz, A./ Hertwig, M. / Pries, L. (2006): Betriebsräte und andere Vertretungsorgane im Vergleich - Strukturen, Arbeitsweisen und Beteiligungsmöglichkeiten, in: WSI-Mitteilungen 59 (9), S. 500-506, https://www.boeckler.de/ wsimit_2006_o9_hauser-ditz.pdf

Kohaut, S. (2019): Tarifbindung: Weiterhin deutliche Unterschiede zwischen Ostund Westdeutschland, in: IAB-Forum vom 22.05.2019, https://www.iab-forum. de/tarifbindung-weiterhin-deutliche-unterschiede-zwischen-ost-und-westdeutschland

\section{AUTOREN}

PETER ELLGUTH ist wissenschaftlicher Mitarbeiter im Institut für Arbeitsmarkt- und Berufsforschung (IAB) der Bundesagentur für Arbeit im Forschungsbereich „Betriebe und Beschäftigung”. Forschungsschwerpunkte: Arbeitsbeziehungen und betriebliche Arbeitszeitpolitik.

\section{peter.ellguth@iab.de}

SUSANNE KOHAUT, Dr., ist wissenschaftliche Mitarbeiterin im IAB im Forschungsbereich „Betriebe und Beschäftigung". Forschungsschwerpunkte: Arbeitsbeziehungen und Betriebsgründungen.

susanne.kohaut@iab.de 